Acta Crystallographica Section E

Structure Reports

Online

ISSN 1600-5368

\section{$N$-[(2-Chlorophenyl)sulfonyl]-2-methoxy- benzamide}

S. Sreenivasa, ${ }^{a}$ B. S. Palakshamurthy, ${ }^{\text {b }}$ E Suresha, ${ }^{c}$ J. Tonannavar, ${ }^{\text {d }}$ Yenagi Jayashree $^{d}$ and P. A. Suchetan ${ }^{\mathrm{e} *}$

a Department of Studies and Research in Chemistry, Tumkur University, Tumkur, Karnataka 572 103, India, 'bepartment of Studies and Research in Physics, U.C.S., Tumkur University, Tumkur, Karnataka 572 103, India, ' University College of Science, Tumkur University, Tumkur 572 103, Karnataka, India, dDepartment of Physics, Karnatak University, Dharwad, Karnataka 580 003, India, and ${ }^{\mathbf{e}}$ Department of Studies and Research in Chemistry, U.C.S., Tumkur University, Tumkur, Karnataka 572 103, India

Correspondence e-mail: pasuchetan@yahoo.co.in

Received 16 October 2013; accepted 22 October 2013

Key indicators: single-crystal X-ray study; $T=293 \mathrm{~K}$; mean $\sigma(\mathrm{C}-\mathrm{C})=0.003 \AA$; $R$ factor $=0.052 ; w R$ factor $=0.163 ;$ data-to-parameter ratio $=25.1$.

The title compound, $\mathrm{C}_{14} \mathrm{H}_{12} \mathrm{ClNO}_{4} \mathrm{~S}$, crystallizes with two molecules in the asymmetric unit. The dihedral angles between the benzene rings are 89.68 (1) (molecule 1) and $82.9(1)^{\circ}$ (molecule 2 ). In each molecule, intramolecular $\mathrm{N}-$ $\mathrm{H}$... O hydrogen bonds between the amide $\mathrm{H}$ atom and the methoxy $\mathrm{O}$ atom generate $S(6)$ loops. In the crystal, molecule 2 is linked into inversion dimers through pairs of $\mathrm{C}-\mathrm{H} \cdots \mathrm{O}$ interactions, forming an $R_{2}^{2}(8)$ ring motif. Molecules 1 and 2 are further linked along the $b$-axis direction through $\mathrm{C}-$ $\mathrm{H} \cdots \pi$ interactions. The crystal structure is further stabilized by several $\pi-\pi$ stacking interactions [centroid-centroid separations $=3.7793$ (1), 3.6697 (1) and 3.6958 (1) ̊]], thus generating a three-dimensional architecture.

\section{Related literature}

For similar structures, see: Gowda et al. (2010); Suchetan et al. (2010a,b, 2013). For hydrogen-bond motifs see: Bernstein et al. (1995).

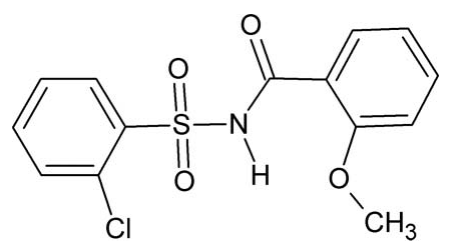

\section{Experimental}

Crystal data

$\mathrm{C}_{14} \mathrm{H}_{12} \mathrm{ClNO}_{4} \mathrm{~S}$

$M_{r}=325.76$

Triclinic, $P \overline{1}$ $a=8.0508$ (3) ̊

$b=12.9487$ (4) $\AA$

$c=14.1915(5) \AA$ $\alpha=83.897(2)^{\circ}$
$\beta=89.368(2)^{\circ}$
$\gamma=89.704(2)^{\circ}$
$V=1470.94(9) \AA^{3}$
$Z=4$

Data collection

Bruker APEXII CCD

diffractometer

34023 measured reflections

Refinement

$R\left[F^{2}>2 \sigma\left(F^{2}\right)\right]=0.052$

$w R\left(F^{2}\right)=0.163$

$S=0.90$

9760 reflections

389 parameters

Table 1

Hydrogen-bond geometry $\left(\AA,{ }^{\circ}\right)$.

$\mathrm{Cg}$ is the centroid of the $\mathrm{C} 22-\mathrm{C} 27$ ring.

\begin{tabular}{|c|c|c|c|c|}
\hline$D-\mathrm{H} \cdots A$ & $D-\mathrm{H}$ & $\mathrm{H} \cdots A$ & $D \cdots A$ & $D-\mathrm{H} \cdots A$ \\
\hline $\mathrm{N} 1-\mathrm{H} N 1 \cdots \mathrm{O} 4$ & $0.84(2)$ & $1.97(2)$ & $2.625(2)$ & $135(2)$ \\
\hline $\mathrm{N} 2-\mathrm{H} N 2 \cdots \mathrm{O} 8$ & $0.83(2)$ & $1.99(2)$ & $2.629(3)$ & $133(2)$ \\
\hline $\mathrm{C} 13-\mathrm{H} 13 \cdots \mathrm{O}^{\mathrm{i}}$ & 0.93 & 2.50 & $3.292(3)$ & 143 \\
\hline $\mathrm{C} 10-\mathrm{H} 10 \cdots C g$ & 0.93 & 2.85 & $3.729(3)$ & 157 \\
\hline
\end{tabular}

Symmetry code: (i) $-x+1,-y+2,-z+1$.

Data collection: APEX2 (Bruker, 2009); cell refinement: APEX2 and SAINT-Plus (Bruker, 2009); data reduction: SAINT-Plus and XPREP (Bruker, 2009); program(s) used to solve structure: SHELXS97 (Sheldrick, 2008); program(s) used to refine structure: SHELXL97 (Sheldrick, 2008); molecular graphics: Mercury (Macrae et al., 2008); software used to prepare material for publication: SHELXL97.

PAS thanks the University Grants Commission (UGC), India, for financial support under its Minor Research Project scheme. JT thanks the Department of Science and Technology (DST), New Delhi, for the SCXRD facility under the PURSE Grant (SR/S9/Z-23/2008/11, 2009) at USIC, Karnatak University.

Supplementary data and figures for this paper are available from the IUCr electronic archives (Reference: SJ5359).

\section{References}

Bernstein, J., Davis, R. E., Shimoni, L. \& Chang, N.-L. (1995). Angew. Chem. Int. Ed. Engl. 34, 1555-1573.

Bruker (2009). APEX2, SAINT-Plus and XPREP. Bruker AXS Inc., Madison, Wisconsin, USA.

Gowda, B. T., Foro, S., Suchetan, P. A. \& Fuess, H. (2010). Acta Cryst. E66, 0794.

Macrae, C. F., Bruno, I. J., Chisholm, J. A., Edgington, P. R., McCabe, P., Pidcock, E., Rodriguez-Monge, L., Taylor, R., van de Streek, J. \& Wood, P. A. (2008). J. Appl. Cryst. 41, 466-470.

Sheldrick, G. M. (2008). Acta Cryst. A64, 112-122.

Suchetan, P. A., Gowda, B. T., Foro, S. \& Fuess, H. (2010a). Acta Cryst. E66, o1040.

Suchetan, P. A., Gowda, B. T., Foro, S. \& Fuess, H. (2010b). Acta Cryst. E66, o1281.

Suchetan, P. A., Palakshamurthy, B. S., Mamatha, G. R., Kumar, V., Mohan, N. R. \& Sreenivasa, S. (2013). Acta Cryst. E69, o1215. $\mu=0.42 \mathrm{~mm}^{-1}$

$T=293 \mathrm{~K}$

9760 independent reflections 6518 reflections with $I>2 \sigma(I)$ $R_{\text {int }}=0.028$

$\mathrm{H}$ atoms treated by a mixture of independent and constrained $\Delta \rho_{\max }=0.68{\mathrm{e} \AA^{-3}}^{-3}$

$\Delta \rho_{\min }=-0.59{\mathrm{e} \AA^{-3}}^{-3}$
Mo $K \alpha$ radiation 


\section{supporting information}

Acta Cryst. (2013). E69, o1716 [doi:10.1107/S1600536813029012]

\section{$N$-[(2-Chlorophenyl)sulfonyl]-2-methoxybenzamide}

\section{S. Sreenivasa, B. S. Palakshamurthy, E Suresha, J. Tonannavar, Yenagi Jayashree and P. A. Suchetan}

\section{S1. Comment}

As a part of our continued efforts to study the crystal structures of $N$-(aroyl)-arylsulfonamides (Suchetan et al., 2010a,b,2013), we report here the crystal structure of the title compound (I) (Fig 1).

The title compound (I) crystallizes with two molecules in the asymmetric unit. This is in contrast to the single molecules observed in the asymmetric units of $N$-(benzoyl)-2-chloro-benzenesulfonamide (II) (Gowda et al., 2010), $N$-(2-chlorobenzoyl)-2-chloro-benzenesulfonamide (III) (Suchetan et al., 2010a), $N$-(2-methylbenzoyl)-2-chloro-benzenesulfonamide (IV) (Suchetan et al., 2010b) and N-(3-methoxybenzoyl)-2-chloro-benzenesulfonamide (V) (Suchetan et al., 2013). In the compound, the conformation of the $\mathrm{N}-\mathrm{H}$ bond in the $\mathrm{C}-\mathrm{SO}_{2}-\mathrm{NH}-\mathrm{C}(\mathrm{O})$ segment is anti to the $\mathrm{C}=\mathrm{O}$ bond. The dihedral angles between the two benzene rings in (I) are $89.68(1)^{\circ}$ (molecule 1) and $82.9(1)^{\circ}$ (molecule 2). Compared to this, the dihedral angles are $73.3(1)^{\circ}$ in II (Gowda et al., 2010), $76.9(1)^{\circ}$ in III (Suchetan et al., 2010a), $78.7(1)^{\circ}$ in IV (Suchetan et al., 2010b) and $87.4(1)^{\circ}$ in V (Suchetan et al., 2013). The conformation of the $\mathrm{N}-\mathrm{H}$ bond is syn to both the $o$-chloro and $o$-methoxy substituents in (I), similar to that observed in V (Suchetan et al., 2013). However, in III (Suchetan et al., 2010a) and IV (Suchetan et al., 2010b) the opposite effect is observed i.e., the $\mathrm{N}-\mathrm{H}$ bond is anti to both the $o$-chloro and $o$-methoxy substituents. In both molecules, intramolecular $\mathrm{N} 1-\mathrm{HN} 1 \cdots \mathrm{O} 4$ and $\mathrm{N} 2-\mathrm{HN} 2 \cdots \mathrm{O} 8$ hydrogen bonds between the amide $\mathrm{H}$ atoms and the methoxy $\mathrm{O}$ atoms, generate $\mathrm{S}(6)$ loops (Bernstein et al., 1995)(Fig 2).

In the crystal, molecule 2 is linked into inversion dimers through intermolecular $\mathrm{C} 13-\mathrm{H} 13 \cdots \mathrm{O} 3$ (Fig 3) interactions forming an $R_{2}{ }^{2}(8)$ ring motif (Bernstein et al., 1995). Molecule 1 and 2 are further linked through $\mathrm{C} 10-\mathrm{H} 10 \cdots \pi$ interactions along the $b$ axis (Fig 4). The crystal structure is further stabilized by several $\pi-\pi$ interactions [centroidcentroid separation being 3.7793 (1) $\AA$ (for $C g 1 — C g 1$ ), 3.6697 (1) $\AA$ (for $C g 3-C g 3$ ) and 3.6958 (1) $\AA$ (for $C g 2-C g 2$ )] (Fig 5). $C g 1$ and $C g 3$ are the centroids of the $\mathrm{C} 8 \cdots \mathrm{C} 13$ and $\mathrm{C} 22 \cdots \mathrm{C} 27$ methoxy benzene rings and $C g 2$ is the centroid of the $\mathrm{C} 1 \cdots \mathrm{C} 6$ sulfonamide ring.

\section{S2. Experimental}

The title compound was prepared by refluxing a mixture of 2-methoxybenzoic acid, 2-chlorobenzene sulfonamide and phosphorous oxychloride for $2 \mathrm{~h}$ on a water bath. The resulting mixture was cooled and poured into ice cold water. The Solid obtained was filtered and washed thoroughly with water and then dissolved in sodium bicarbonate solution. The compound was later reprecipitated by acidifying the filtered solution with dilute $\mathrm{HCl}$. The filtered and dried solid was recrystallized to the constant melting point ( $429 \mathrm{~K}$ ). Colorless prisms of (I) were obtained from a slow evaporation of an ethanolic solution at room temperature. 


\section{S3. Refinement}

The $\mathrm{H}$ atoms of the $\mathrm{NH}$ groups were located in a difference map and later refined freely. The other $\mathrm{H}$ atoms were positioned with idealized geometry using a riding model with $\mathrm{C}-\mathrm{H}=0.93-0.96 \AA$. All $\mathrm{H}$ atoms were refined with isotropic displacement parameters (set to $1.2-1.5$ times of the $U_{\mathrm{eq}}$ of the parent atom).

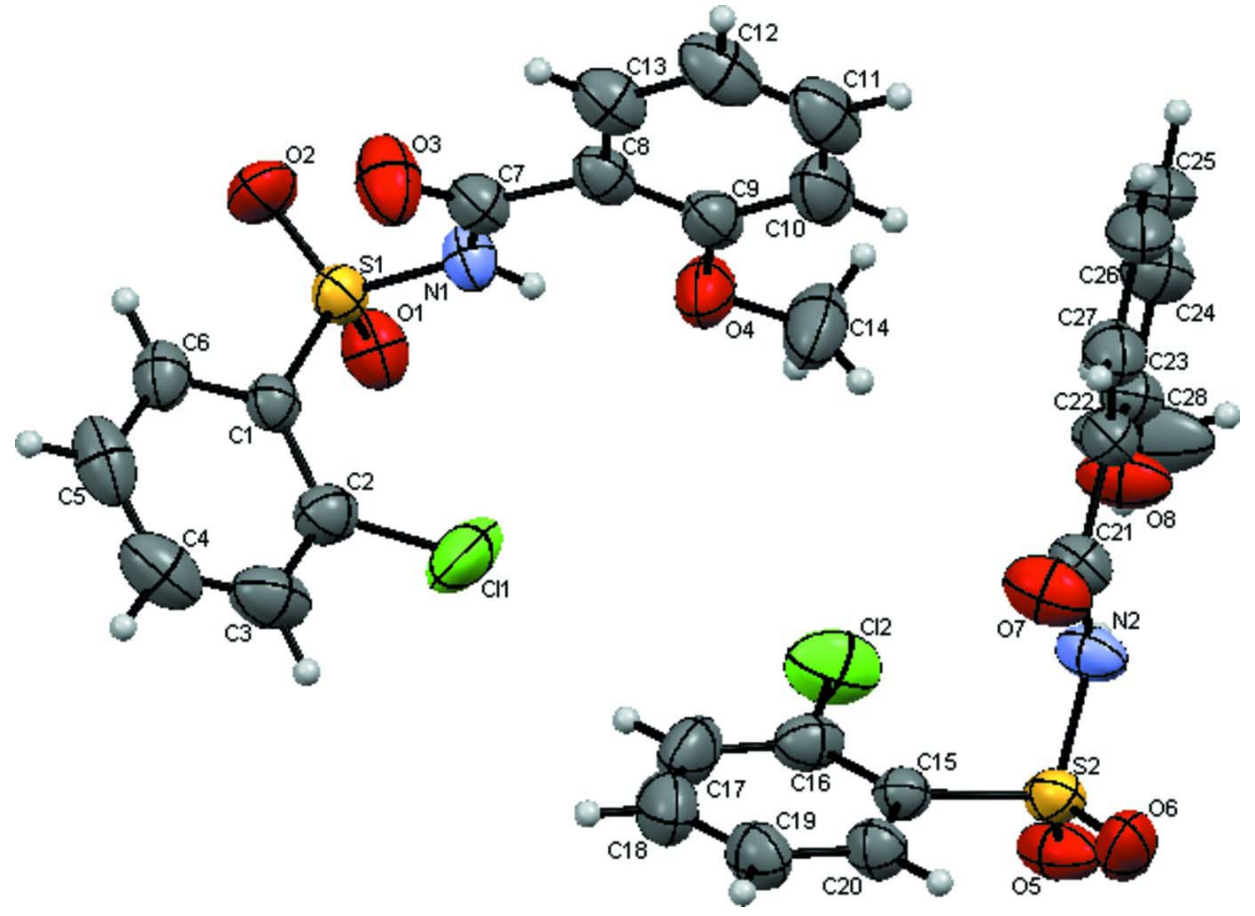

\section{Figure 1}

Molecular structure of the title compound, showing the atom-labeling scheme. Displacement ellipsoids are drawn at the $50 \%$ probability level. 


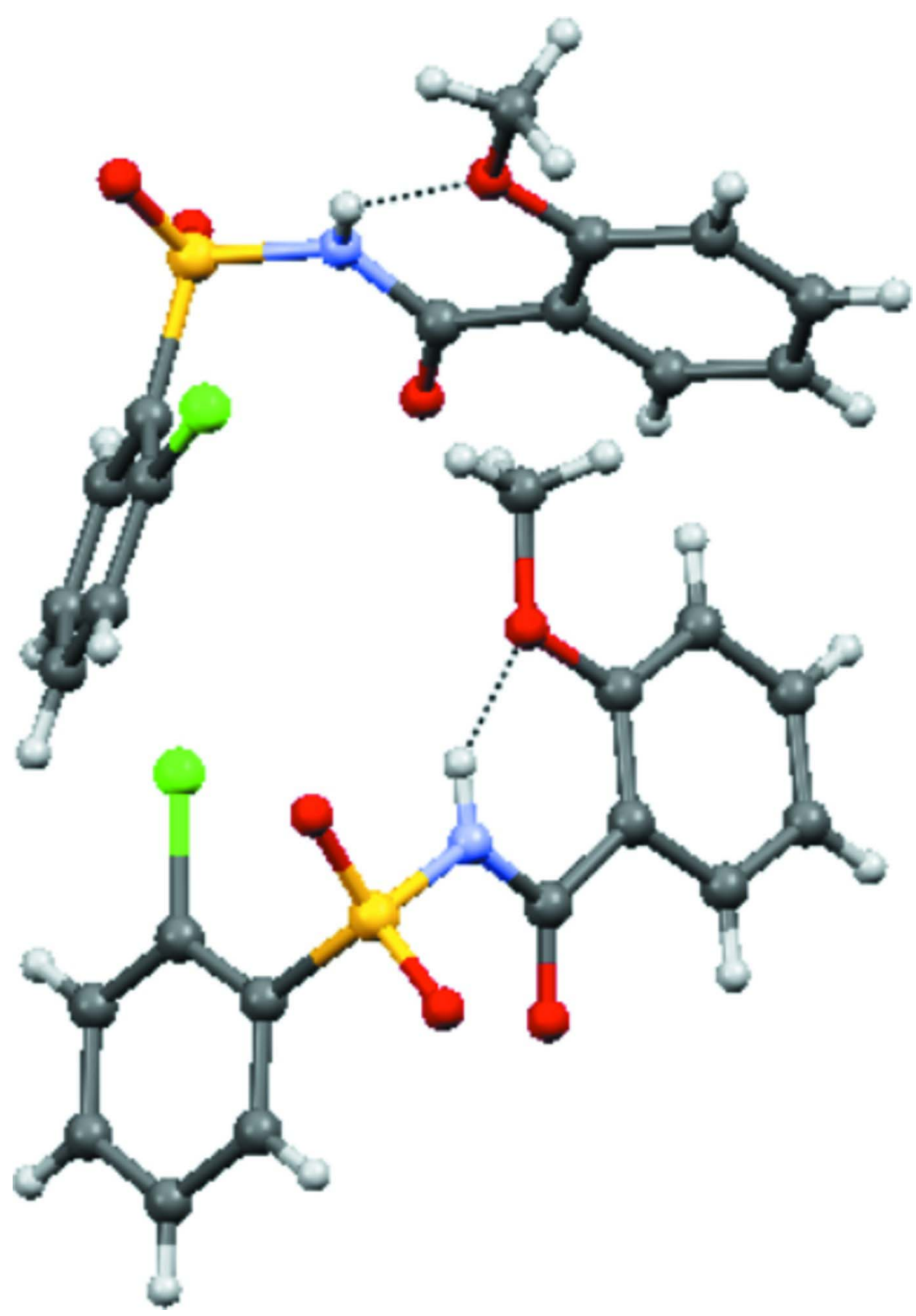

Figure 2

Formation of intramolecular $\mathrm{N}-\mathrm{H} \cdots \mathrm{O}$ hydrogen bonds, dashed lines, generating $S(6)$ loops. 


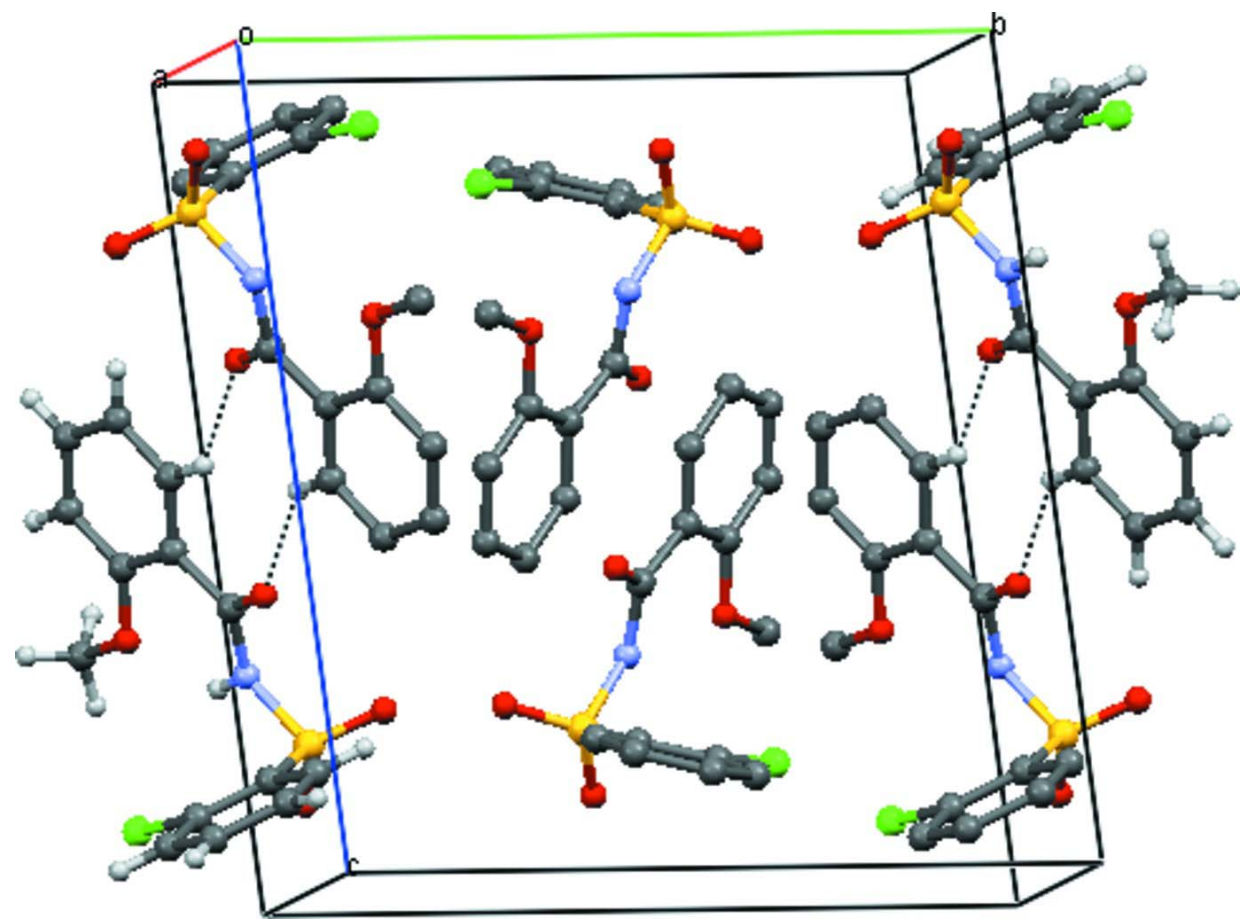

Figure 3

Packing of molecules in I through intermolecular $\mathrm{C}-\mathrm{H} \cdots \mathrm{O}$ interactions, dashed lines, generating $R_{2}^{2}(8)$ loops. $\mathrm{H}$ atoms not involved in H-bonding are ommitted for clarity. 


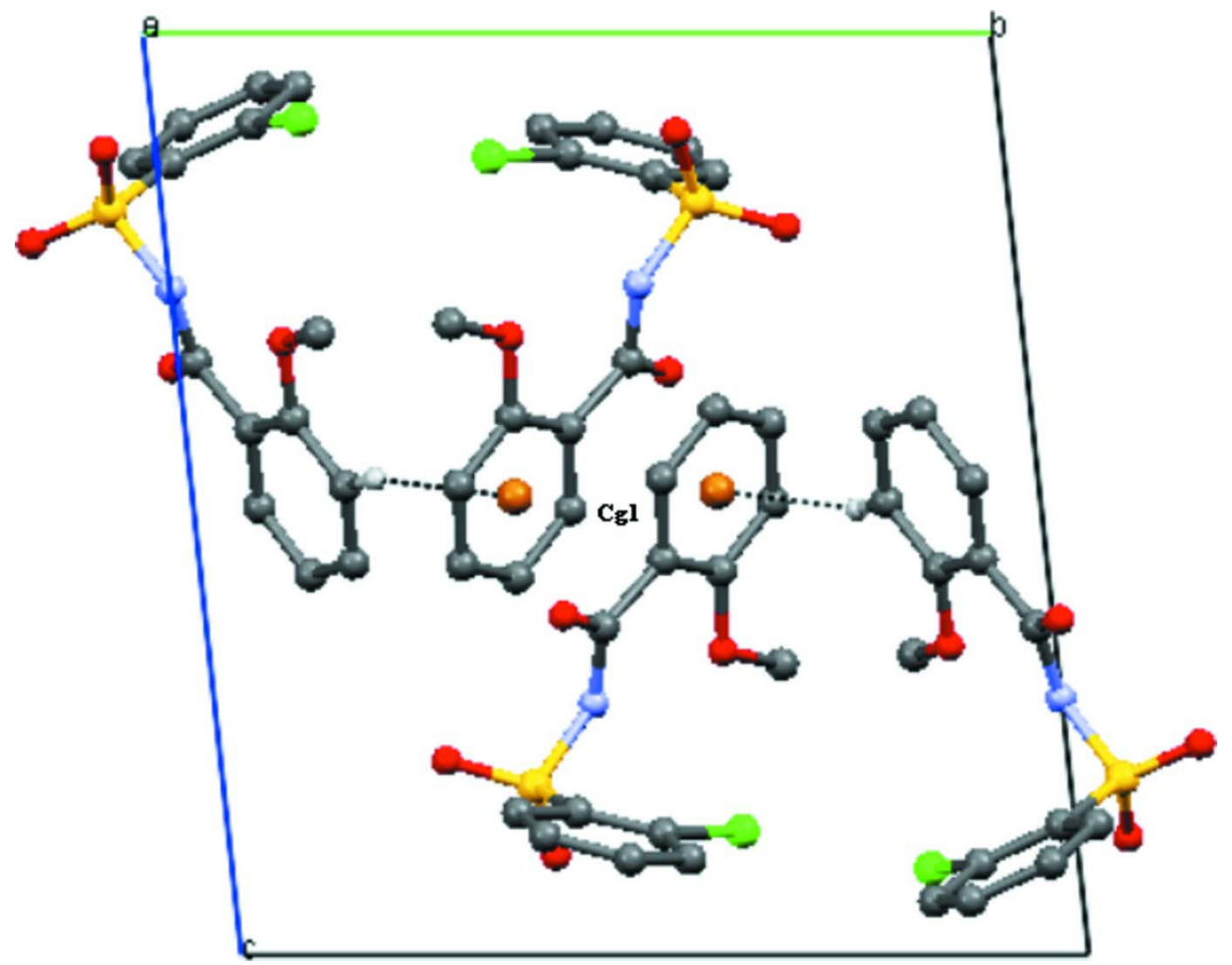

\section{Figure 4}

Linking of molecules along $b$ axis in (I) through $\mathrm{C}-\mathrm{H} \cdots \mathrm{Cg} 1$ interactions. $\mathrm{H}$ atoms not involved in $\mathrm{H}$-bonding are ommitted for clarity. 


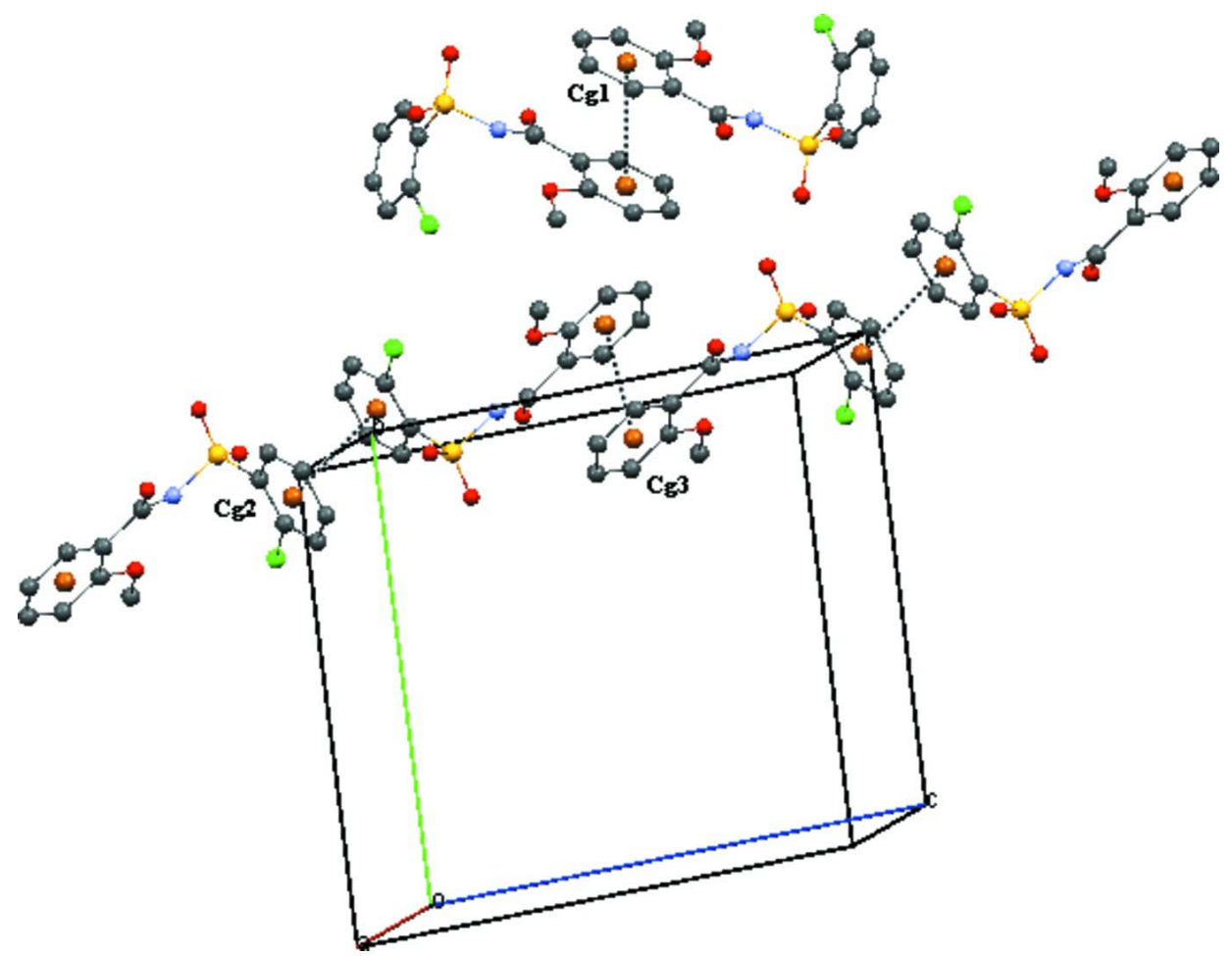

Figure 5

Stacking of molecules in I, through $C g \cdots C g$ interactions. Where $C g 1$ and $C g 3$ are the centroids of the $\mathrm{C} 8-\mathrm{C} 13$ and $\mathrm{C} 22$ $-\mathrm{C} 27$ methoxy benzene rings and $\mathrm{Cg} 2$ is the centroid of the $\mathrm{C} 1-\mathrm{C} 6$ sulfonamide ring. $\mathrm{H}$ atoms are ommitted for clarity.

\section{$N$-[(2-Chlorophenyl)sulfonyl]-2-methoxybenzamide}

Crystal data

$\mathrm{C}_{14} \mathrm{H}_{12} \mathrm{ClNO}_{4} \mathrm{~S}$

$M_{r}=325.76$

Triclinic, $P \overline{1}$

Hall symbol: -P 1

$a=8.0508(3) \AA$

$b=12.9487$ (4) $\AA$

$c=14.1915(5) \AA$

$\alpha=83.897(2)^{\circ}$

$\beta=89.368(2)^{\circ}$

$\gamma=89.704(2)^{\circ}$

$V=1470.94(9) \AA^{3}$

$Z=4$

\section{Data collection}

\section{Bruker APEXII CCD}

diffractometer

Radiation source: fine-focus sealed tube

Graphite monochromator

$\varphi$ and $\omega$ scans

34023 measured reflections

9760 independent reflections
$F(000)=672$

Prism

$D_{\mathrm{x}}=1.471 \mathrm{Mg} \mathrm{m}^{-3}$

Melting point: $429 \mathrm{~K}$

Mo $K \alpha$ radiation, $\lambda=0.71073 \AA$

Cell parameters from 1234 reflections

$\theta=1.6-31.8^{\circ}$

$\mu=0.42 \mathrm{~mm}^{-1}$

$T=293 \mathrm{~K}$

Prism, colourless

$0.36 \times 0.29 \times 0.23 \mathrm{~mm}$

6518 reflections with $I>2 \sigma(I)$

$R_{\text {int }}=0.028$

$\theta_{\text {max }}=31.8^{\circ}, \theta_{\text {min }}=1.6^{\circ}$

$h=-11 \rightarrow 11$

$k=-17 \rightarrow 19$

$l=-20 \rightarrow 20$ 


\section{Refinement}

Refinement on $F^{2}$

Least-squares matrix: full

$R\left[F^{2}>2 \sigma\left(F^{2}\right)\right]=0.052$

$w R\left(F^{2}\right)=0.163$

$S=0.90$

9760 reflections

389 parameters

0 restraints

Primary atom site location: structure-invariant direct methods
Secondary atom site location: difference Fourier map

Hydrogen site location: inferred from neighbouring sites

$\mathrm{H}$ atoms treated by a mixture of independent and constrained refinement

$w=1 /\left[\sigma^{2}\left(F_{\mathrm{o}}^{2}\right)+(0.0876 P)^{2}+0.587 P\right]$ where $P=\left(F_{\mathrm{o}}{ }^{2}+2 F_{\mathrm{c}}{ }^{2}\right) / 3$

$(\Delta / \sigma)_{\max }=0.002$

$\Delta \rho_{\max }=0.68 \mathrm{e} \AA^{-3}$

$\Delta \rho_{\min }=-0.59 \mathrm{e} \AA^{-3}$

\section{Special details}

Geometry. All s.u.'s (except the s.u. in the dihedral angle between two 1.s. planes) are estimated using the full covariance matrix. The cell s.u.'s are taken into account individually in the estimation of s.u.'s in distances, angles and torsion angles; correlations between s.u.'s in cell parameters are only used when they are defined by crystal symmetry. An approximate (isotropic) treatment of cell s.u.'s is used for estimating s.u.'s involving l.s. planes.

Refinement. Refinement of $F^{2}$ against ALL reflections. The weighted $R$-factor $w R$ and goodness of fit $S$ are based on $F^{2}$, conventional $R$-factors $R$ are based on $F$, with $F$ set to zero for negative $F^{2}$. The threshold expression of $F^{2}>2 \sigma\left(F^{2}\right)$ is used only for calculating $R$-factors(gt) $e t c$. and is not relevant to the choice of reflections for refinement. $R$-factors based on $F^{2}$ are statistically about twice as large as those based on $F$, and $R$ - factors based on ALL data will be even larger.

Fractional atomic coordinates and isotropic or equivalent isotropic displacement parameters $\left(\AA^{2}\right)$

\begin{tabular}{lllll}
\hline & $x$ & $y$ & $z$ & $U_{\mathrm{iso}} * / U_{\mathrm{eq}}$ \\
\hline C28 & $-0.6577(5)$ & $1.3256(3)$ & $0.3153(3)$ & $0.1291(16)$ \\
H28A & -0.6248 & 1.2553 & 0.3352 & $0.194^{*}$ \\
H28B & -0.6856 & 1.3319 & 0.2494 & $0.194^{*}$ \\
H28C & -0.7526 & 1.3435 & 0.3519 & $0.194^{*}$ \\
HN2 & $-0.408(3)$ & $1.5138(18)$ & $0.2590(15)$ & $0.057(6)^{*}$ \\
HN1 & $0.080(3)$ & $1.0299(18)$ & $0.2691(15)$ & $0.056(6)^{*}$ \\
C1 & $0.4099(2)$ & $1.01693(13)$ & $0.14113(11)$ & $0.0413(3)$ \\
C2 & $0.3854(3)$ & $1.11730(15)$ & $0.09867(13)$ & $0.0526(4)$ \\
C3 & $0.5167(3)$ & $1.17506(18)$ & $0.05849(16)$ & $0.0692(6)$ \\
H3 & 0.4993 & 1.2427 & 0.0311 & $0.083^{*}$ \\
C4 & $0.6705(4)$ & $1.1329(2)$ & $0.05921(16)$ & $0.0732(7)$ \\
H4 & 0.7584 & 1.1720 & 0.0317 & $0.088^{*}$ \\
C5 & $0.7001(3)$ & $1.0329(2)$ & $0.09992(17)$ & $0.0696(7)$ \\
H5 & 0.8066 & 1.0047 & 0.0995 & $0.084^{*}$ \\
C6 & $0.5675(2)$ & $0.97436(17)$ & $0.14195(14)$ & $0.0533(4)$ \\
H6 & 0.5857 & 0.9072 & 0.1702 & $0.064^{*}$ \\
C7 & $0.2633(3)$ & $1.02032(16)$ & $0.35657(13)$ & $0.0504(4)$ \\
C8 & $0.1764(2)$ & $1.07678(14)$ & $0.42977(12)$ & $0.0476(4)$ \\
C9 & $0.0234(3)$ & $1.12861(14)$ & $0.41869(13)$ & $0.0520(4)$ \\
C10 & $-0.0391(3)$ & $1.18159(17)$ & $0.49168(16)$ & $0.0654(6)$ \\
H10 & -0.1393 & 1.2174 & 0.4840 & $0.078^{*}$ \\
C11 & $0.0456(3)$ & $1.18137(18)$ & $0.57482(17)$ & $0.0701(6)$ \\
H11 & 0.0022 & 1.2170 & 0.6231 & $0.084^{*}$ \\
C12 & $0.1934(3)$ & $1.1294(2)$ & $0.58768(16)$ & $0.0699(6)$
\end{tabular}




\begin{tabular}{|c|c|c|c|c|}
\hline $\mathrm{H} 12$ & 0.2495 & 1.1285 & 0.6447 & $0.084 *$ \\
\hline $\mathrm{C} 13$ & $0.2585(3)$ & $1.07799(18)$ & $0.51452(14)$ & 0.0593 \\
\hline $\mathrm{H} 13$ & 0.3598 & 1.0436 & 0.5228 & $0.071^{*}$ \\
\hline C14 & $-0.2212(4)$ & $1.1690(3)$ & $0.3257(2)$ & $0.0902(9)$ \\
\hline H14A & -0.2893 & 1.1400 & 0.3777 & $0.135^{*}$ \\
\hline H14B & -0.2682 & 1.1528 & 0.2671 & $0.135^{*}$ \\
\hline $\mathrm{H} 14 \mathrm{C}$ & -0.2159 & 1.2430 & 0.3260 & $0.135^{*}$ \\
\hline $\mathrm{C} 15$ & $-0.0676(2)$ & $1.58600(13)$ & $0.15496(11)$ & $0.0407(3)$ \\
\hline $\mathrm{C} 16$ & $-0.0377(3)$ & $1.48635(14)$ & $0.13150(13)$ & $0.0529(4)$ \\
\hline $\mathrm{C} 17$ & $0.1220(3)$ & $1.45853(18)$ & $0.10457(17)$ & $0.0687(6)$ \\
\hline H17 & 0.1427 & 1.3924 & 0.0872 & $0.082 *$ \\
\hline $\mathrm{C} 18$ & $0.2491(3)$ & $1.5294(2)$ & $0.10383(17)$ & $0.0691(6)$ \\
\hline $\mathrm{H} 18$ & 0.3555 & 1.5105 & 0.0859 & $0.083^{*}$ \\
\hline C19 & $0.2212(3)$ & $1.62651(19)$ & $0.12890(15)$ & $0.0601(5)$ \\
\hline H19 & 0.3083 & 1.6733 & 0.1291 & $0.072 *$ \\
\hline $\mathrm{C} 20$ & $0.0624(2)$ & $1.65527(15)$ & $0.15405(13)$ & 0.0485 \\
\hline $\mathrm{H} 20$ & 0.0429 & 1.7219 & 0.1705 & $0.058^{*}$ \\
\hline $\mathrm{C} 21$ & $-0.2474(2)$ & $1.53203(16)$ & $0.35569(13)$ & $0.0500(4)$ \\
\hline $\mathrm{C} 22$ & $-0.3193(2)$ & $1.45265(15)$ & $0.42864(12)$ & $0.0483(4)$ \\
\hline $\mathrm{C} 23$ & $-0.4520(3)$ & $1.38669(16)$ & $0.41651(13)$ & $0.0527(4)$ \\
\hline $\mathrm{C} 24$ & $-0.5045(3)$ & $1.31545(18)$ & $0.49100(16)$ & $0.0670(6)$ \\
\hline $\mathrm{H} 24$ & -0.5931 & 1.2716 & 0.4825 & $0.080^{*}$ \\
\hline $\mathrm{C} 25$ & $-0.4266(3)$ & $1.3094(2)$ & $0.57675(16)$ & $0.0727(7)$ \\
\hline $\mathrm{H} 25$ & -0.4626 & 1.2614 & 0.6260 & $0.087^{*}$ \\
\hline $\mathrm{C} 26$ & $-0.2968(3)$ & $1.3730(2)$ & $0.59061(15)$ & $0.0703(7)$ \\
\hline $\mathrm{H} 26$ & -0.2450 & 1.3690 & 0.6492 & $0.084^{*}$ \\
\hline $\mathrm{C} 27$ & $-0.2425(3)$ & $1.44394(18)$ & $0.51671(14)$ & $0.0600(5)$ \\
\hline $\mathrm{H} 27$ & -0.1529 & 1.4866 & 0.5261 & $0.072 *$ \\
\hline N1 & $0.1728(2)$ & $1.00103(13)$ & $0.27880(11)$ & 0.0495 \\
\hline $\mathrm{N} 2$ & $-0.3337(2)$ & $1.55288(14)$ & $0.27293(11)$ & $0.0526(4)$ \\
\hline $\mathrm{O} 1$ & $0.11150(18)$ & $0.93899(12)$ & $0.12765(10)$ & $0.0612(4)$ \\
\hline $\mathrm{O} 2$ & $0.3149(2)$ & $0.84221(11)$ & $0.22865(11)$ & $0.0659(4)$ \\
\hline $\mathrm{O} 3$ & $0.4060(2)$ & $0.99214(16)$ & $0.36392(12)$ & $0.0797(5)$ \\
\hline $\mathrm{O} 4$ & $-0.0573(2)$ & $1.12592(13)$ & $0.33491(10)$ & $0.0666(4)$ \\
\hline O5 & $-0.37606(18)$ & $1.61675(14)$ & $0.10764(11)$ & $0.0721(5)$ \\
\hline O6 & $-0.2480(2)$ & $1.73358(11)$ & $0.21074(12)$ & $0.0707(4)$ \\
\hline O7 & $-0.1198(2)$ & $1.57723(14)$ & $0.36765(11)$ & $0.0716(4)$ \\
\hline O8 & $-0.5245(2)$ & $1.39365(13)$ & $0.32949(11)$ & $0.0713(5)$ \\
\hline S1 & $0.24534(6)$ & $0.93882(3)$ & $0.19164(3)$ & $0.04506(12)$ \\
\hline S2 & $-0.26639(6)$ & $1.63258(4)$ & $0.18268(3)$ & $0.04893(13)$ \\
\hline $\mathrm{Cl1}$ & $0.19015(10)$ & $1.17448(5)$ & $0.09374(6)$ & $0.0900(2)$ \\
\hline $\mathrm{Cl} 2$ & $-0.19141(10)$ & $1.39327(5)$ & $0.13476(5)$ & $0.0854(2)$ \\
\hline
\end{tabular}

Atomic displacement parameters $\left(\AA^{2}\right)$

\begin{tabular}{lllllll}
\hline & $U^{11}$ & $U^{22}$ & $U^{33}$ & $U^{12}$ & $U^{13}$ & $U^{23}$ \\
\hline $\mathrm{C} 28$ & $0.132(3)$ & $0.133(3)$ & $0.112(2)$ & $-0.091(3)$ & $-0.049(2)$ & $0.042(2)$ \\
$\mathrm{C} 1$ & $0.0459(9)$ & $0.0403(8)$ & $0.0384(8)$ & $-0.0003(7)$ & $0.0031(7)$ & $-0.0087(6)$
\end{tabular}


supporting information

\begin{tabular}{|c|c|c|c|c|c|c|}
\hline $\mathrm{C} 2$ & $0.0644(12)$ & $0.0439(9)$ & $0.0494(10)$ & $-0.0006(9)$ & $0.0024(9)$ & $-0.0045(7)$ \\
\hline $\mathrm{C} 3$ & $0.0948(18)$ & $0.0555(12)$ & $0.0565(12)$ & $-0.0177(12)$ & $0.0091(12)$ & $-0.0021(9)$ \\
\hline $\mathrm{C} 4$ & $0.0816(17)$ & $0.0851(17)$ & $0.0549(12)$ & $-0.0299(14)$ & $0.0139(11)$ & $-0.0172(11)$ \\
\hline $\mathrm{C} 5$ & $0.0465(11)$ & $0.105(2)$ & $0.0630(13)$ & $-0.0006(12)$ & $0.0042(10)$ & $-0.0339(13)$ \\
\hline C6 & $0.0512(11)$ & $0.0604(12)$ & $0.0502(10)$ & $0.0086(9)$ & $-0.0004(8)$ & $-0.0147(8)$ \\
\hline C7 & $0.0534(11)$ & $0.0536(10)$ & $0.0443(9)$ & $-0.0040(8)$ & $0.0056(8)$ & $-0.0057(7)$ \\
\hline $\mathrm{C} 8$ & $0.0552(11)$ & $0.0433(9)$ & $0.0447(9)$ & $-0.0114(8)$ & $0.0098(8)$ & $-0.0071(7)$ \\
\hline C9 & $0.0661(12)$ & $0.0423(9)$ & $0.0476(9)$ & $-0.0038(8)$ & $0.0121(9)$ & $-0.0061(7)$ \\
\hline $\mathrm{C} 10$ & $0.0828(16)$ & $0.0524(12)$ & $0.0623(12)$ & $0.0013(11)$ & $0.0169(11)$ & $-0.0141(9)$ \\
\hline C11 & $0.0926(18)$ & $0.0607(13)$ & $0.0605(12)$ & $-0.0164(13)$ & $0.0222(12)$ & $-0.0239(10)$ \\
\hline C12 & $0.0870(17)$ & $0.0763(15)$ & $0.0491(11)$ & $-0.0278(13)$ & $0.0061(11)$ & $-0.0179(10)$ \\
\hline $\mathrm{C} 13$ & $0.0635(13)$ & $0.0647(13)$ & $0.0506(10)$ & $-0.0142(10)$ & $0.0051(9)$ & $-0.0105(9)$ \\
\hline $\mathrm{C} 14$ & $0.0921(19)$ & $0.103(2)$ & $0.0768(16)$ & $0.0482(17)$ & $-0.0087(14)$ & $-0.0173(15)$ \\
\hline $\mathrm{C} 15$ & $0.0437(9)$ & $0.0381(8)$ & $0.0395(8)$ & $-0.0020(7)$ & $0.0014(6)$ & $-0.0001(6)$ \\
\hline $\mathrm{C} 16$ & $0.0707(13)$ & $0.0398(9)$ & $0.0477(9)$ & $-0.0062(9)$ & $-0.0028(9)$ & $-0.0021(7)$ \\
\hline $\mathrm{C} 17$ & $0.0887(17)$ & $0.0498(12)$ & $0.0686(13)$ & $0.0219(12)$ & $-0.0008(12)$ & $-0.0119(10)$ \\
\hline $\mathrm{C} 18$ & $0.0566(13)$ & $0.0793(16)$ & $0.0706(14)$ & $0.0147(12)$ & $0.0058(11)$ & $-0.0057(12)$ \\
\hline $\mathrm{C} 19$ & $0.0480(11)$ & $0.0692(14)$ & $0.0620(12)$ & $-0.0037(10)$ & $0.0064(9)$ & $-0.0027(10)$ \\
\hline $\mathrm{C} 20$ & $0.0475(10)$ & $0.0454(9)$ & $0.0525(10)$ & $-0.0065(8)$ & $0.0059(8)$ & $-0.0049(7)$ \\
\hline $\mathrm{C} 21$ & $0.0490(10)$ & $0.0550(11)$ & $0.0457(9)$ & $-0.0053(8)$ & $0.0039(8)$ & $-0.0046(8)$ \\
\hline $\mathrm{C} 22$ & $0.0474(10)$ & $0.0529(10)$ & $0.0431(9)$ & $0.0055(8)$ & $0.0058(7)$ & $0.0007(7)$ \\
\hline $\mathrm{C} 23$ & $0.0539(11)$ & $0.0528(10)$ & $0.0489(10)$ & $0.0000(9)$ & $0.0040(8)$ & $0.0058(8)$ \\
\hline $\mathrm{C} 24$ & $0.0699(14)$ & $0.0591(12)$ & $0.0669(13)$ & $-0.0036(10)$ & $0.0124(11)$ & $0.0152(10)$ \\
\hline $\mathrm{C} 25$ & $0.0782(16)$ & $0.0750(15)$ & $0.0577(12)$ & $0.0167(13)$ & $0.0154(11)$ & $0.0230(11)$ \\
\hline $\mathrm{C} 26$ & $0.0752(15)$ & $0.0866(17)$ & $0.0457(10)$ & $0.0259(13)$ & $0.0012(10)$ & $0.0070(10)$ \\
\hline $\mathrm{C} 27$ & $0.0569(12)$ & $0.0752(14)$ & $0.0471(10)$ & $0.0115(10)$ & $-0.0006(9)$ & $-0.0029(9)$ \\
\hline N1 & $0.0474(9)$ & $0.0572(10)$ & $0.0453(8)$ & $0.0030(8)$ & $0.0056(7)$ & $-0.0122(7)$ \\
\hline $\mathrm{N} 2$ & $0.0454(9)$ & $0.0626(10)$ & $0.0471(8)$ & $-0.0139(8)$ & $0.0024(7)$ & $0.0072(7)$ \\
\hline $\mathrm{O} 1$ & $0.0529(8)$ & $0.0724(10)$ & $0.0619(8)$ & $-0.0076(7)$ & $0.0000(7)$ & $-0.0233(7)$ \\
\hline $\mathrm{O} 2$ & $0.0877(11)$ & $0.0367(7)$ & $0.0720(9)$ & $0.0038(7)$ & $0.0076(8)$ & $0.0002(6)$ \\
\hline $\mathrm{O} 3$ & $0.0606(10)$ & $0.1193(15)$ & $0.0633(9)$ & $0.0189(10)$ & $-0.0076(8)$ & $-0.0295(9)$ \\
\hline $\mathrm{O} 4$ & $0.0737(10)$ & $0.0727(10)$ & $0.0544(8)$ & $0.0239(8)$ & $0.0005(7)$ & $-0.0131(7)$ \\
\hline O5 & $0.0499(8)$ & $0.0969(12)$ & $0.0621(9)$ & $-0.0079(8)$ & $-0.0072(7)$ & $0.0262(8)$ \\
\hline O6 & $0.0714(10)$ & $0.0454(8)$ & $0.0932(11)$ & $0.0081(7)$ & $0.0246(9)$ & $-0.0007(7)$ \\
\hline $\mathrm{O} 7$ & $0.0663(10)$ & $0.0863(11)$ & $0.0612(9)$ & $-0.0286(9)$ & $-0.0060(7)$ & $-0.0011(8)$ \\
\hline $\mathrm{O} 8$ & $0.0776(11)$ & $0.0732(10)$ & $0.0592(8)$ & $-0.0338(8)$ & $-0.0117(7)$ & $0.0142(7)$ \\
\hline S1 & 0.0499 (3) & $0.0389(2)$ & $0.0469(2)$ & $-0.00208(18)$ & $0.00520(18)$ & $-0.00783(16)$ \\
\hline S2 & $0.0410(2)$ & $0.0494(3)$ & $0.0531(2)$ & $-0.00109(18)$ & $0.00462(18)$ & $0.00939(18)$ \\
\hline $\mathrm{Cl1}$ & $0.0898(5)$ & $0.0554(3)$ & $0.1200(6)$ & $0.0259(3)$ & $-0.0007(4)$ & $0.0116(3)$ \\
\hline $\mathrm{Cl} 2$ & $0.1187(6)$ & $0.0528(3)$ & $0.0861(4)$ & $-0.0362(3)$ & $0.0009(4)$ & $-0.0118(3)$ \\
\hline
\end{tabular}

Geometric parameters ( $\left.\AA,{ }^{\circ}\right)$

\begin{tabular}{llll}
\hline $\mathrm{C} 28-\mathrm{O} 8$ & $1.421(3)$ & $\mathrm{C} 15-\mathrm{C} 20$ & $1.381(2)$ \\
$\mathrm{C} 28-\mathrm{H} 28 \mathrm{~A}$ & 0.9600 & $\mathrm{C} 15-\mathrm{C} 16$ & $1.386(3)$ \\
$\mathrm{C} 28-\mathrm{H} 28 \mathrm{~B}$ & 0.9600 & $\mathrm{C} 15-\mathrm{S} 2$ & $1.7626(18)$ \\
$\mathrm{C} 28-\mathrm{H} 28 \mathrm{C}$ & 0.9600 & $\mathrm{C} 16-\mathrm{C} 17$ & $1.394(3)$ \\
$\mathrm{C} 1-\mathrm{C} 6$ & $1.381(3)$ & $\mathrm{C} 16-\mathrm{C} 12$ & $1.729(2)$
\end{tabular}




\begin{tabular}{|c|c|c|c|}
\hline $\mathrm{C} 1-\mathrm{C} 2$ & $1.387(3)$ & $\mathrm{C} 17-\mathrm{C} 18$ & $1.377(4)$ \\
\hline $\mathrm{C} 1-\mathrm{S} 1$ & $1.7692(18)$ & $\mathrm{C} 17-\mathrm{H} 17$ & 0.9300 \\
\hline $\mathrm{C} 2-\mathrm{C} 3$ & $1.381(3)$ & $\mathrm{C} 18-\mathrm{C} 19$ & $1.360(3)$ \\
\hline $\mathrm{C} 2-\mathrm{Cl1}$ & $1.733(2)$ & $\mathrm{C} 18-\mathrm{H} 18$ & 0.9300 \\
\hline $\mathrm{C} 3-\mathrm{C} 4$ & $1.350(4)$ & $\mathrm{C} 19-\mathrm{C} 20$ & $1.383(3)$ \\
\hline $\mathrm{C} 3-\mathrm{H} 3$ & 0.9300 & $\mathrm{C} 19-\mathrm{H} 19$ & 0.9300 \\
\hline $\mathrm{C} 4-\mathrm{C} 5$ & $1.381(4)$ & $\mathrm{C} 20-\mathrm{H} 20$ & 0.9300 \\
\hline $\mathrm{C} 4-\mathrm{H} 4$ & 0.9300 & $\mathrm{C} 21-\mathrm{O} 7$ & $1.208(2)$ \\
\hline $\mathrm{C} 5-\mathrm{C} 6$ & $1.403(3)$ & $\mathrm{C} 21-\mathrm{N} 2$ & $1.372(2)$ \\
\hline $\mathrm{C} 5-\mathrm{H} 5$ & 0.9300 & $\mathrm{C} 21-\mathrm{C} 22$ & $1.493(3)$ \\
\hline $\mathrm{C} 6-\mathrm{H} 6$ & 0.9300 & $\mathrm{C} 22-\mathrm{C} 27$ & $1.394(3)$ \\
\hline $\mathrm{C} 7-\mathrm{O} 3$ & $1.206(2)$ & $\mathrm{C} 22-\mathrm{C} 23$ & $1.394(3)$ \\
\hline $\mathrm{C} 7-\mathrm{N} 1$ & $1.375(3)$ & $\mathrm{C} 23-\mathrm{O} 8$ & $1.366(2)$ \\
\hline $\mathrm{C} 7-\mathrm{C} 8$ & $1.498(3)$ & $\mathrm{C} 23-\mathrm{C} 24$ & $1.391(3)$ \\
\hline $\mathrm{C} 8-\mathrm{C} 13$ & $1.380(3)$ & $\mathrm{C} 24-\mathrm{C} 25$ & $1.370(3)$ \\
\hline $\mathrm{C} 8-\mathrm{C} 9$ & $1.402(3)$ & $\mathrm{C} 24-\mathrm{H} 24$ & 0.9300 \\
\hline $\mathrm{C} 9-\mathrm{O} 4$ & $1.365(2)$ & $\mathrm{C} 25-\mathrm{C} 26$ & $1.363(4)$ \\
\hline $\mathrm{C} 9-\mathrm{C} 10$ & $1.390(3)$ & $\mathrm{C} 25-\mathrm{H} 25$ & 0.9300 \\
\hline $\mathrm{C} 10-\mathrm{C} 11$ & $1.369(3)$ & $\mathrm{C} 26-\mathrm{C} 27$ & $1.387(3)$ \\
\hline $\mathrm{C} 10-\mathrm{H} 10$ & 0.9300 & $\mathrm{C} 26-\mathrm{H} 26$ & 0.9300 \\
\hline $\mathrm{C} 11-\mathrm{C} 12$ & $1.368(4)$ & $\mathrm{C} 27-\mathrm{H} 27$ & 0.9300 \\
\hline $\mathrm{C} 11-\mathrm{H} 11$ & 0.9300 & $\mathrm{~N} 1-\mathrm{S} 1$ & $1.6464(16)$ \\
\hline $\mathrm{C} 12-\mathrm{C} 13$ & $1.388(3)$ & $\mathrm{N} 1-\mathrm{HN} 1$ & $0.84(2)$ \\
\hline $\mathrm{C} 12-\mathrm{H} 12$ & 0.9300 & $\mathrm{~N} 2-\mathrm{S} 2$ & $1.6456(16)$ \\
\hline $\mathrm{C} 13-\mathrm{H} 13$ & 0.9300 & $\mathrm{~N} 2-\mathrm{HN} 2$ & $0.83(2)$ \\
\hline $\mathrm{C} 14-\mathrm{O} 4$ & $1.431(3)$ & $\mathrm{O} 1-\mathrm{S} 1$ & $1.4167(15)$ \\
\hline $\mathrm{C} 14-\mathrm{H} 14 \mathrm{~A}$ & 0.9600 & $\mathrm{O} 2-\mathrm{S} 1$ & $1.4193(15)$ \\
\hline C14-H14B & 0.9600 & $\mathrm{O} 5-\mathrm{S} 2$ & $1.4229(16)$ \\
\hline $\mathrm{C} 14-\mathrm{H} 14 \mathrm{C}$ & 0.9600 & $\mathrm{O} 6-\mathrm{S} 2$ & $1.4159(17)$ \\
\hline $\mathrm{O} 8-\mathrm{C} 28-\mathrm{H} 28 \mathrm{~A}$ & 109.5 & $\mathrm{C} 15-\mathrm{C} 16-\mathrm{Cl} 2$ & $122.57(17)$ \\
\hline $\mathrm{O} 8-\mathrm{C} 28-\mathrm{H} 28 \mathrm{~B}$ & 109.5 & $\mathrm{C} 17-\mathrm{C} 16-\mathrm{Cl} 2$ & $117.90(16)$ \\
\hline $\mathrm{H} 28 \mathrm{~A}-\mathrm{C} 28-\mathrm{H} 28 \mathrm{~B}$ & 109.5 & $\mathrm{C} 18-\mathrm{C} 17-\mathrm{C} 16$ & $119.7(2)$ \\
\hline $\mathrm{O} 8-\mathrm{C} 28-\mathrm{H} 28 \mathrm{C}$ & 109.5 & $\mathrm{C} 18-\mathrm{C} 17-\mathrm{H} 17$ & 120.1 \\
\hline $\mathrm{H} 28 \mathrm{~A}-\mathrm{C} 28-\mathrm{H} 28 \mathrm{C}$ & 109.5 & $\mathrm{C} 16-\mathrm{C} 17-\mathrm{H} 17$ & 120.1 \\
\hline $\mathrm{H} 28 \mathrm{~B}-\mathrm{C} 28-\mathrm{H} 28 \mathrm{C}$ & 109.5 & $\mathrm{C} 19-\mathrm{C} 18-\mathrm{C} 17$ & $121.0(2)$ \\
\hline $\mathrm{C} 6-\mathrm{C} 1-\mathrm{C} 2$ & $119.26(18)$ & $\mathrm{C} 19-\mathrm{C} 18-\mathrm{H} 18$ & 119.5 \\
\hline $\mathrm{C} 6-\mathrm{C} 1-\mathrm{S} 1$ & $118.13(14)$ & $\mathrm{C} 17-\mathrm{C} 18-\mathrm{H} 18$ & 119.5 \\
\hline $\mathrm{C} 2-\mathrm{C} 1-\mathrm{S} 1$ & $122.58(14)$ & $\mathrm{C} 18-\mathrm{C} 19-\mathrm{C} 20$ & $119.6(2)$ \\
\hline $\mathrm{C} 3-\mathrm{C} 2-\mathrm{C} 1$ & $120.8(2)$ & $\mathrm{C} 18-\mathrm{C} 19-\mathrm{H} 19$ & 120.2 \\
\hline $\mathrm{C} 3-\mathrm{C} 2-\mathrm{Cl} 1$ & $117.68(17)$ & $\mathrm{C} 20-\mathrm{C} 19-\mathrm{H} 19$ & 120.2 \\
\hline $\mathrm{C} 1-\mathrm{C} 2-\mathrm{C} 11$ & $121.52(15)$ & $\mathrm{C} 15-\mathrm{C} 20-\mathrm{C} 19$ & $120.67(19)$ \\
\hline $\mathrm{C} 4-\mathrm{C} 3-\mathrm{C} 2$ & $119.7(2)$ & $\mathrm{C} 15-\mathrm{C} 20-\mathrm{H} 20$ & 119.7 \\
\hline $\mathrm{C} 4-\mathrm{C} 3-\mathrm{H} 3$ & 120.2 & $\mathrm{C} 19-\mathrm{C} 20-\mathrm{H} 20$ & 119.7 \\
\hline $\mathrm{C} 2-\mathrm{C} 3-\mathrm{H} 3$ & 120.2 & $\mathrm{O} 7-\mathrm{C} 21-\mathrm{N} 2$ & $120.38(18)$ \\
\hline $\mathrm{C} 3-\mathrm{C} 4-\mathrm{C} 5$ & $121.4(2)$ & $\mathrm{O} 7-\mathrm{C} 21-\mathrm{C} 22$ & $122.59(18)$ \\
\hline $\mathrm{C} 3-\mathrm{C} 4-\mathrm{H} 4$ & 119.3 & $\mathrm{~N} 2-\mathrm{C} 21-\mathrm{C} 22$ & $117.03(17)$ \\
\hline $\mathrm{C} 5-\mathrm{C} 4-\mathrm{H} 4$ & 119.3 & $\mathrm{C} 27-\mathrm{C} 22-\mathrm{C} 23$ & $117.87(18)$ \\
\hline
\end{tabular}




\begin{tabular}{|c|c|c|c|}
\hline $\mathrm{C} 4-\mathrm{C} 5-\mathrm{C} 6$ & $119.1(2)$ & $\mathrm{C} 27-\mathrm{C} 22-\mathrm{C} 21$ & $115.59(18)$ \\
\hline $\mathrm{C} 4-\mathrm{C} 5-\mathrm{H} 5$ & 120.4 & $\mathrm{C} 23-\mathrm{C} 22-\mathrm{C} 21$ & $126.54(17)$ \\
\hline $\mathrm{C} 6-\mathrm{C} 5-\mathrm{H} 5$ & 120.4 & $\mathrm{O} 8-\mathrm{C} 23-\mathrm{C} 24$ & $122.1(2)$ \\
\hline $\mathrm{C} 1-\mathrm{C} 6-\mathrm{C} 5$ & $119.7(2)$ & $\mathrm{O} 8-\mathrm{C} 23-\mathrm{C} 22$ & $117.77(16)$ \\
\hline $\mathrm{C} 1-\mathrm{C} 6-\mathrm{H} 6$ & 120.1 & $\mathrm{C} 24-\mathrm{C} 23-\mathrm{C} 22$ & $120.1(2)$ \\
\hline $\mathrm{C} 5-\mathrm{C} 6-\mathrm{H} 6$ & 120.1 & $\mathrm{C} 25-\mathrm{C} 24-\mathrm{C} 23$ & $120.4(2)$ \\
\hline $\mathrm{O} 3-\mathrm{C} 7-\mathrm{N} 1$ & $120.28(18)$ & $\mathrm{C} 25-\mathrm{C} 24-\mathrm{H} 24$ & 119.8 \\
\hline $\mathrm{O} 3-\mathrm{C} 7-\mathrm{C} 8$ & $122.73(18)$ & $\mathrm{C} 23-\mathrm{C} 24-\mathrm{H} 24$ & 119.8 \\
\hline $\mathrm{N} 1-\mathrm{C} 7-\mathrm{C} 8$ & $116.99(17)$ & $\mathrm{C} 26-\mathrm{C} 25-\mathrm{C} 24$ & $120.7(2)$ \\
\hline $\mathrm{C} 13-\mathrm{C} 8-\mathrm{C} 9$ & $118.47(18)$ & $\mathrm{C} 26-\mathrm{C} 25-\mathrm{H} 25$ & 119.7 \\
\hline $\mathrm{C} 13-\mathrm{C} 8-\mathrm{C} 7$ & $115.62(18)$ & $\mathrm{C} 24-\mathrm{C} 25-\mathrm{H} 25$ & 119.7 \\
\hline $\mathrm{C} 9-\mathrm{C} 8-\mathrm{C} 7$ & $125.90(17)$ & $\mathrm{C} 25-\mathrm{C} 26-\mathrm{C} 27$ & $119.5(2)$ \\
\hline $\mathrm{O} 4-\mathrm{C} 9-\mathrm{C} 10$ & $122.6(2)$ & $\mathrm{C} 25-\mathrm{C} 26-\mathrm{H} 26$ & 120.3 \\
\hline $\mathrm{O} 4-\mathrm{C} 9-\mathrm{C} 8$ & $117.88(16)$ & $\mathrm{C} 27-\mathrm{C} 26-\mathrm{H} 26$ & 120.3 \\
\hline $\mathrm{C} 10-\mathrm{C} 9-\mathrm{C} 8$ & $119.5(2)$ & $\mathrm{C} 26-\mathrm{C} 27-\mathrm{C} 22$ & $121.4(2)$ \\
\hline $\mathrm{C} 11-\mathrm{C} 10-\mathrm{C} 9$ & $120.5(2)$ & $\mathrm{C} 26-\mathrm{C} 27-\mathrm{H} 27$ & 119.3 \\
\hline $\mathrm{C} 11-\mathrm{C} 10-\mathrm{H} 10$ & 119.8 & $\mathrm{C} 22-\mathrm{C} 27-\mathrm{H} 27$ & 119.3 \\
\hline $\mathrm{C} 9-\mathrm{C} 10-\mathrm{H} 10$ & 119.8 & $\mathrm{C} 7-\mathrm{N} 1-\mathrm{S} 1$ & $124.23(14)$ \\
\hline $\mathrm{C} 12-\mathrm{C} 11-\mathrm{C} 10$ & $120.8(2)$ & $\mathrm{C} 7-\mathrm{N} 1-\mathrm{HN} 1$ & $119.7(15)$ \\
\hline $\mathrm{C} 12-\mathrm{C} 11-\mathrm{H} 11$ & 119.6 & $\mathrm{~S} 1-\mathrm{N} 1-\mathrm{HN} 1$ & $115.3(15)$ \\
\hline $\mathrm{C} 10-\mathrm{C} 11-\mathrm{H} 11$ & 119.6 & $\mathrm{C} 21-\mathrm{N} 2-\mathrm{S} 2$ & $123.34(14)$ \\
\hline $\mathrm{C} 11-\mathrm{C} 12-\mathrm{C} 13$ & $119.1(2)$ & $\mathrm{C} 21-\mathrm{N} 2-\mathrm{HN} 2$ & $120.8(15)$ \\
\hline $\mathrm{C} 11-\mathrm{C} 12-\mathrm{H} 12$ & 120.4 & $\mathrm{~S} 2-\mathrm{N} 2-\mathrm{HN} 2$ & $113.6(15)$ \\
\hline $\mathrm{C} 13-\mathrm{C} 12-\mathrm{H} 12$ & 120.4 & $\mathrm{C} 9-\mathrm{O} 4-\mathrm{C} 14$ & $118.77(17)$ \\
\hline $\mathrm{C} 8-\mathrm{C} 13-\mathrm{C} 12$ & $121.5(2)$ & $\mathrm{C} 23-\mathrm{O} 8-\mathrm{C} 28$ & $118.52(18)$ \\
\hline $\mathrm{C} 8-\mathrm{C} 13-\mathrm{H} 13$ & 119.2 & $\mathrm{O} 1-\mathrm{S} 1-\mathrm{O} 2$ & $118.75(10)$ \\
\hline $\mathrm{C} 12-\mathrm{C} 13-\mathrm{H} 13$ & 119.2 & $\mathrm{O} 1-\mathrm{S} 1-\mathrm{N} 1$ & $104.48(9)$ \\
\hline $\mathrm{O} 4-\mathrm{C} 14-\mathrm{H} 14 \mathrm{~A}$ & 109.5 & $\mathrm{O} 2-\mathrm{S} 1-\mathrm{N} 1$ & $109.88(9)$ \\
\hline $\mathrm{O} 4-\mathrm{C} 14-\mathrm{H} 14 \mathrm{~B}$ & 109.5 & $\mathrm{O} 1-\mathrm{S} 1-\mathrm{C} 1$ & $110.61(9)$ \\
\hline $\mathrm{H} 14 \mathrm{~A}-\mathrm{C} 14-\mathrm{H} 14 \mathrm{~B}$ & 109.5 & $\mathrm{O} 2-\mathrm{S} 1-\mathrm{C} 1$ & $107.38(9)$ \\
\hline $\mathrm{O} 4-\mathrm{C} 14-\mathrm{H} 14 \mathrm{C}$ & 109.5 & $\mathrm{~N} 1-\mathrm{S} 1-\mathrm{C} 1$ & $104.90(8)$ \\
\hline $\mathrm{H} 14 \mathrm{~A}-\mathrm{C} 14-\mathrm{H} 14 \mathrm{C}$ & 109.5 & $\mathrm{O} 6-\mathrm{S} 2-\mathrm{O} 5$ & $119.47(11)$ \\
\hline $\mathrm{H} 14 \mathrm{~B}-\mathrm{C} 14-\mathrm{H} 14 \mathrm{C}$ & 109.5 & $\mathrm{O} 6-\mathrm{S} 2-\mathrm{N} 2$ & $109.83(10)$ \\
\hline $\mathrm{C} 20-\mathrm{C} 15-\mathrm{C} 16$ & $119.46(17)$ & $\mathrm{O} 5-\mathrm{S} 2-\mathrm{N} 2$ & $104.50(9)$ \\
\hline $\mathrm{C} 20-\mathrm{C} 15-\mathrm{S} 2$ & $116.91(14)$ & $\mathrm{O} 6-\mathrm{S} 2-\mathrm{C} 15$ & $108.02(9)$ \\
\hline $\mathrm{C} 16-\mathrm{C} 15-\mathrm{S} 2$ & $123.60(14)$ & $\mathrm{O} 5-\mathrm{S} 2-\mathrm{C} 15$ & $108.78(9)$ \\
\hline $\mathrm{C} 15-\mathrm{C} 16-\mathrm{C} 17$ & $119.53(19)$ & $\mathrm{N} 2-\mathrm{S} 2-\mathrm{C} 15$ & $105.37(8)$ \\
\hline $\mathrm{C} 6-\mathrm{C} 1-\mathrm{C} 2-\mathrm{C} 3$ & $-0.8(3)$ & $\mathrm{O} 7-\mathrm{C} 21-\mathrm{C} 22-\mathrm{C} 23$ & $-170.5(2)$ \\
\hline $\mathrm{S} 1-\mathrm{C} 1-\mathrm{C} 2-\mathrm{C} 3$ & $-178.80(16)$ & $\mathrm{N} 2-\mathrm{C} 21-\mathrm{C} 22-\mathrm{C} 23$ & $9.9(3)$ \\
\hline $\mathrm{C} 6-\mathrm{C} 1-\mathrm{C} 2-\mathrm{Cl} 1$ & $179.04(14)$ & $\mathrm{C} 27-\mathrm{C} 22-\mathrm{C} 23-\mathrm{O} 8$ & $-178.48(18)$ \\
\hline $\mathrm{S} 1-\mathrm{C} 1-\mathrm{C} 2-\mathrm{Cl} 1$ & $1.0(2)$ & $\mathrm{C} 21-\mathrm{C} 22-\mathrm{C} 23-\mathrm{O} 8$ & $0.5(3)$ \\
\hline $\mathrm{C} 1-\mathrm{C} 2-\mathrm{C} 3-\mathrm{C} 4$ & $1.0(3)$ & $\mathrm{C} 27-\mathrm{C} 22-\mathrm{C} 23-\mathrm{C} 24$ & $0.3(3)$ \\
\hline $\mathrm{C} 11-\mathrm{C} 2-\mathrm{C} 3-\mathrm{C} 4$ & $-178.82(18)$ & $\mathrm{C} 21-\mathrm{C} 22-\mathrm{C} 23-\mathrm{C} 24$ & $179.3(2)$ \\
\hline $\mathrm{C} 2-\mathrm{C} 3-\mathrm{C} 4-\mathrm{C} 5$ & $-0.4(4)$ & $\mathrm{O} 8-\mathrm{C} 23-\mathrm{C} 24-\mathrm{C} 25$ & $178.7(2)$ \\
\hline $\mathrm{C} 3-\mathrm{C} 4-\mathrm{C} 5-\mathrm{C} 6$ & $-0.5(3)$ & $\mathrm{C} 22-\mathrm{C} 23-\mathrm{C} 24-\mathrm{C} 25$ & $0.0(3)$ \\
\hline $\mathrm{C} 2-\mathrm{C} 1-\mathrm{C} 6-\mathrm{C} 5$ & $-0.1(3)$ & $\mathrm{C} 23-\mathrm{C} 24-\mathrm{C} 25-\mathrm{C} 26$ & $0.1(4)$ \\
\hline $\mathrm{S} 1-\mathrm{C} 1-\mathrm{C} 6-\mathrm{C} 5$ & $178.03(15)$ & $\mathrm{C} 24-\mathrm{C} 25-\mathrm{C} 26-\mathrm{C} 27$ & $-0.5(4)$ \\
\hline
\end{tabular}




$\begin{array}{llll}\mathrm{C} 4-\mathrm{C} 5-\mathrm{C} 6-\mathrm{C} 1 & 0.7(3) & \mathrm{C} 25-\mathrm{C} 26-\mathrm{C} 27-\mathrm{C} 22 & 0.9(3) \\ \mathrm{O} 3-\mathrm{C} 7-\mathrm{C} 8-\mathrm{C} 13 & -11.6(3) & \mathrm{C} 23-\mathrm{C} 22-\mathrm{C} 27-\mathrm{C} 26 & -0.8(3) \\ \mathrm{N} 1-\mathrm{C} 7-\mathrm{C} 8-\mathrm{C} 13 & 168.32(17) & \mathrm{C} 21-\mathrm{C} 22-\mathrm{C} 27-\mathrm{C} 26 & -179.84(19) \\ \mathrm{O} 3-\mathrm{C} 7-\mathrm{C} 8-\mathrm{C} 9 & 167.3(2) & \mathrm{O} 3-\mathrm{C} 7-\mathrm{N} 1-\mathrm{S} 1 & 0.8(3) \\ \mathrm{N} 1-\mathrm{C} 7-\mathrm{C} 8-\mathrm{C} 9 & -12.8(3) & \mathrm{C} 8-\mathrm{C} 7-\mathrm{N} 1-\mathrm{S} 1 & -179.16(13) \\ \mathrm{C} 13-\mathrm{C} 8-\mathrm{C} 9-\mathrm{O} 4 & -179.34(17) & \mathrm{O} 7-\mathrm{C} 21-\mathrm{N} 2-\mathrm{S} 2 & 4.3(3) \\ \mathrm{C} 7-\mathrm{C} 8-\mathrm{C} 9-\mathrm{O} 4 & 1.8(3) & \mathrm{C} 22-\mathrm{C} 21-\mathrm{N} 2-\mathrm{S} 2 & -176.06(14) \\ \mathrm{C} 13-\mathrm{C} 8-\mathrm{C} 9-\mathrm{C} 10 & 1.6(3) & \mathrm{C} 10-\mathrm{C} 9-\mathrm{O} 4-\mathrm{C} 14 & -7.3(3) \\ \mathrm{C} 7-\mathrm{C} 8-\mathrm{C} 9-\mathrm{C} 10 & -177.29(18) & \mathrm{C} 8-\mathrm{C} 9-\mathrm{O} 4-\mathrm{C} 14 & 173.6(2) \\ \mathrm{O} 4-\mathrm{C} 9-\mathrm{C} 10-\mathrm{C} 11 & 179.4(2) & \mathrm{C} 24-\mathrm{C} 23-\mathrm{O}-\mathrm{C} 28 & 0.0(4) \\ \mathrm{C} 8-\mathrm{C} 9-\mathrm{C} 10-\mathrm{C} 11 & -1.5(3) & \mathrm{C} 22-\mathrm{C} 23-\mathrm{O}-\mathrm{C} 28 & 178.8(3) \\ \mathrm{C} 9-\mathrm{C} 10-\mathrm{C} 11-\mathrm{C} 12 & 0.2(3) & \mathrm{C} 7-\mathrm{N} 1-\mathrm{S} 1-\mathrm{O} 1 & -179.03(16) \\ \mathrm{C} 10-\mathrm{C} 11-\mathrm{C} 12-\mathrm{C} 13 & 1.1(4) & \mathrm{C} 7-\mathrm{N} 1-\mathrm{S} 1-\mathrm{O} 2 & 52.54(18) \\ \mathrm{C} 9-\mathrm{C} 8-\mathrm{C} 13-\mathrm{C} 12 & -0.3(3) & \mathrm{C} 7-\mathrm{N} 1-\mathrm{S} 1-\mathrm{C} 1 & -62.61(17) \\ \mathrm{C} 7-\mathrm{C} 8-\mathrm{C} 13-\mathrm{C} 12 & 178.66(19) & \mathrm{C} 6-\mathrm{C} 1-\mathrm{S} 1-\mathrm{O} 1 & -128.77(15) \\ \mathrm{C} 11-\mathrm{C} 12-\mathrm{C} 13-\mathrm{C} 8 & -1.0(3) & \mathrm{C} 2-\mathrm{C} 1-\mathrm{S} 1-\mathrm{O} 1 & 49.27(17) \\ \mathrm{C} 20-\mathrm{C} 15-\mathrm{C} 16-\mathrm{C} 17 & -1.9(3) & \mathrm{C} 6-\mathrm{C} 1-\mathrm{S} 1-\mathrm{O} 2 & 2.24(17) \\ \mathrm{S} 2-\mathrm{C} 15-\mathrm{C} 16-\mathrm{C} 17 & 175.80(15) & \mathrm{C} 2-\mathrm{C} 1-\mathrm{S} 1-\mathrm{O} 2 & -179.72(15) \\ \mathrm{C} 20-\mathrm{C} 15-\mathrm{C} 16-\mathrm{C} 12 & 177.69(14) & \mathrm{C} 6-\mathrm{C} 1-\mathrm{S} 1-\mathrm{N} 1 & 119.12(15) \\ \mathrm{S} 2-\mathrm{C} 15-\mathrm{C} 16-\mathrm{C} 2 & -4.6(2) & \mathrm{C} 2-\mathrm{C} 1-\mathrm{S} 1-\mathrm{N} 1 & -62.85(17) \\ \mathrm{C} 15-\mathrm{C} 16-\mathrm{C} 17-\mathrm{C} 18 & 1.6(3) & \mathrm{C} 21-\mathrm{N} 2-\mathrm{S} 2-\mathrm{O} 6 & -61.0(2) \\ \mathrm{C} 12-\mathrm{C} 16-\mathrm{C} 17-\mathrm{C} 18 & -178.03(18) & \mathrm{C} 21-\mathrm{N} 2-\mathrm{S} 2-\mathrm{O} 5 & 169.72(18) \\ \mathrm{C} 16-\mathrm{C} 17-\mathrm{C} 18-\mathrm{C} 19 & -0.1(4) & \mathrm{C} 21-\mathrm{N} 2-\mathrm{S} 2-\mathrm{C} 15 & 55.13(19) \\ \mathrm{C} 17-\mathrm{C} 18-\mathrm{C} 19-\mathrm{C} 20 & -1.1(3) & \mathrm{C} 20-\mathrm{C} 15-\mathrm{S} 2-\mathrm{O} 6 & -7.14(17) \\ \mathrm{C} 16-\mathrm{C} 15-\mathrm{C} 20-\mathrm{C} 19 & 0.8(3) & \mathrm{C} 16-\mathrm{C} 15-\mathrm{S} 2-\mathrm{O} 6 & 175.07(15) \\ \mathrm{S} 2-\mathrm{C} 15-\mathrm{C} 20-\mathrm{C} 19 & -177.10(15) & \mathrm{C} 20-\mathrm{C} 15-\mathrm{S} 2-\mathrm{O} 5 & 123.94(15) \\ \mathrm{C} 18-\mathrm{C} 19-\mathrm{C} 20-\mathrm{C} 15 & 0.7(3) & \mathrm{C} 16-\mathrm{C} 15-\mathrm{S} 2-\mathrm{O} 5 & -12.85(17) \\ \mathrm{O} 7-\mathrm{C} 21-\mathrm{C} 22-\mathrm{C} 27 & 8.5(3) & \mathrm{C} 20-\mathrm{C} 15-\mathrm{S} 2-\mathrm{N} 2 & 57.73(17) \\ \mathrm{N} 2-\mathrm{C} 21-\mathrm{C} 22-\mathrm{C} 27 & -171.13(18) & \mathrm{C} 16-\mathrm{C} 15-\mathrm{S} 2-\mathrm{N} 2 & \\ & & & \end{array}$

Hydrogen-bond geometry $\left(A,{ }^{\circ}\right)$

$\mathrm{Cg}$ is the centroid of the $\mathrm{C} 22-\mathrm{C} 27$ ring.

\begin{tabular}{lllll}
\hline$D-\mathrm{H} \cdots A$ & $D-\mathrm{H}$ & $\mathrm{H} \cdots A$ & $D \cdots A$ & $D-\mathrm{H} \cdots A$ \\
\hline $\mathrm{N} 1-\mathrm{H} N 1 \cdots \mathrm{O} 4$ & $0.84(2)$ & $1.97(2)$ & $2.625(2)$ & $135(2)$ \\
$\mathrm{N} 2-\mathrm{H} N 2 \cdots \mathrm{O} 8$ & $0.83(2)$ & $1.99(2)$ & $2.629(3)$ & $133(2)$ \\
$\mathrm{C} 13-\mathrm{H} 13 \cdots \mathrm{O} 3$ & & & \\
$\mathrm{C} 10-\mathrm{H} 10 \cdots \mathrm{Cg}$ & 0.93 & 2.50 & $3.292(3)$ & 143 \\
& 0.93 & 2.85 & $3.729(3)$ & 157 \\
\hline
\end{tabular}

Symmetry code: (i) $-x+1,-y+2,-z+1$. 